\title{
Reversible Computation in Wireless Communications
}

\author{
Harun $\operatorname{Siljak}^{(\bowtie)}$ \\ CONNECT Centre, Trinity College, The University of Dublin, Dublin, Ireland \\ harun.siljak@tcd.ie
}

\begin{abstract}
This chapter presents pioneering work in applying reversible computation paradigms to wireless communications. These applications range from developing reversible hardware architectures for underwater acoustic communications to novel distributed optimisation procedures in large radio-frequency antenna arrays based on reversing Petri nets. Throughout the chapter, we discuss the rationale for introducing reversible computation in the domain of wireless communications, exploring the inherently reversible properties of communication channels and systems formed by devices in a wireless network.
\end{abstract}

\section{Introduction}

Wireless communication systems come in different shapes and sizes: from radio frequency $(\mathrm{RF})$ systems we use in everyday life, to underwater acoustic communications (UAC) used where RF attenuation prevents use of radio communications. These two examples are of interest to this case study, as we explored the potential role of reversible computation in improving modern wireless communications in the RF and acoustic domains.

In the RF context, we examine the concept of distributed massive MIMO (multiple input multiple output) systems. The distributed massive MIMO paradigm will have an increasing relevance in fifth generation $(5 \mathrm{G})$ wireless systems and post-5G era, as it will allow formerly centralised base stations to operate as a group of hundreds (thousands) of small antennas distributed in space, serving many users by beamforming the signal to them, operating using distributed algorithms hence providing reduced power consumption and reduced computational overhead. Our aim is to explore the application of reversible computation paradigms in such systems to contribute in additional reduction of power consumption, but also to help in fault recovery and meaningful undoing of algorithmic steps in control and optimisation of such systems.

In the underwater acoustic context, we recognised the wave time reversal scheme as a physical example of reversibility, a physical method waiting for its reversible circuit implementation. The mechanism of wave time reversal is analogous to reversible computation as we know it, and as such it admits elegant and simple circuit implementation benefiting from all reversible computation advantages. With this inherent reversibility in mind, we take the question of wave 
time reversal in underwater conditions a step further, and ask about realistic models of such systems using reversible computation paradigms, and investigate the options of controlling the environment in which this process is used for communication.

Communication is inherently reversible: the communication channel changes direction all the time, with the transmitter and the receiver changing roles and transmitting through the same medium. Modulation and demodulation, coding and decoding all these processes aim for information conservation and reversibility. Hence the motivation for this study is clear: can reversible computation help in achieving goals of modern wireless communication: increasing access, decreasing latency and power consumption, minimising information losses?

In this chapter, we present results on optimisation schemes for massive MIMO based on reversing Petri nets, reversible hardware for wave time reversal, and some preliminary thoughts on our work in progress on modelling and control of wave time reversal in reversible cellular automata, as well as control of these automata in general.

\section{Reversing Petri Nets and Massive MIMO}

\subsection{The Problem}

In the distributed massive MIMO system described in the previous section, not all antennas need to be active at all times. Selecting a subset of antennas to operate at a particular time instant allows the system to retain advantages of a large antenna array, including interference suppression, spatial multiplexing and diversity [16] while reducing the number of radio frequency (RF) chains and the number of antennas to power [13]. The computational demand of optimal transmit antenna selection for large antenna arrays [11] makes it impractical, suggesting the necessity of suboptimal approaches. Traditionally, these approaches were centralised and based on the knowledge of the communication channel between every user and every antenna in the array; one widely used algorithm is the greedy algorithm [12] which operates iteratively by adding the antenna that increases the sum rate the most when joined with the set of already selected antennas. In decentralised algorithms similar procedures are conducted on much smaller subsets of antennas [21], leading to similar results in overall performance. Our approach here is decentralised, and it relies on Reversing Petri nets (RPN) [17] as the underlying paradigm. As this chapter focuses on applications, the reader interested in details about reversing Petri nets used in this example is advised to see [18]. The presentation here is based on [22].

The optimisation problem we are solving is downlink (transmit) antenna selection of $N_{T S}$ antennas at the distributed massive MIMO base station with $N_{T}$ antennas, in presence of $N_{R}$ single antenna users. We maximise the sumcapacity

$$
\mathcal{C}=\max _{\mathbf{P}, \mathbf{H}_{c}} \log _{2} \operatorname{det}\left(\mathbf{I}+\rho \frac{N_{R}}{N_{T S}} \mathbf{H}_{c} \mathbf{P} \mathbf{H}_{c}^{H}\right)
$$


where $\rho$ is the signal to noise ratio $(\mathrm{SNR}), \mathbf{I}$ a $N_{T S} \times N_{T S}$ identity matrix, $\mathbf{P}$ a diagonal $N_{R} \times N_{R}$ power distribution matrix. $\mathbf{H}_{c}$ is the $N_{T S} \times N_{R}$ channel submatrix for a selected subset of antennas from the $N_{T} \times N_{R}$ channel matrix $\mathbf{H}[10]$.

In the case of receiver antenna selection, addition of any antenna to the set of selected antennas improves the overall sum-capacity, as its equivalent of Eq. (1) does not involve scaling by the number of selected antennas (i.e. there is not a power budget to be distributed over antennas in the receive case). This problem is submodular and has a guaranteed (suboptimal) performance bound for the previously described greedy algorithm. The greedy algorithm does not have performance bound for the transmitter antenna selection, as the case described by Eq. (1) does not fulfil the submodularity condition [24]; the addition of an antenna to the already selected set of antennas can decrease channel capacity.

As done in $[21,24]$, we optimise (1) with two variables, the subset of selected antennas and the optimal power distribution over them successively: first, $\mathbf{P}$ is fixed to having all diagonal elements equal to $1 / N_{R}$ (total power is equal to $\left.\rho N_{R} / N_{T S}\right)$, and after the antenna selection $\mathbf{P}$ is optimised by the water filling algorithm for zero forcing.

Figure 1 illustrates the proposed algorithm based on RPN: the antennas are Petri net places (circles $\mathrm{A}-\mathrm{G}$ ), with the token (bright circle) in a place indicating that the current state of the algorithm asks for that place (that antenna) to be on. The places are divided into overlapping neighbourhoods $\left(N_{1}\right.$ and $N_{2}$ in our toy example) and each two adjacent places have a common neighbourhood. Transitions between places move tokens around based on the sum capacity calculations, with rules described below:

1. A transition is possible if there is a token in exactly one of the two places (e.g. B and G in Fig. 1) it connects. Otherwise (e.g. A and B, or E and F) it is not possible.

2. The enabled transition will occur if the sum capacity (1) calculated for all antennas with a token in the neighbourhood shared by the two places (for B and $\mathrm{G}$, that is neighbourhood $N_{1}$ ) is less than the sum capacity calculated for the same neighbourhood, but with the token moved to the empty place (in case of B-G transition, this means $\mathcal{C}_{A B}<\mathcal{C}_{A G}$, sum-capacity of antennas $A$ and $B$ is smaller than that of $A$ and $G$ ). Otherwise, it does not occur.

3. In case of several possible transitions from one place (A-E, A-D, A-C) the one with the greatest sum-capacity difference (i.e. improvement) has the priority.

4. There is no designated order in transition execution, and transitions are performed until a stable state is reached.

The algorithm starts from a configuration of $n$ tokens in random places and converges to a stable final configuration in a small number (in our experiments, up to five) of iterations (passes) through the whole network. As the RPN conserves the number of tokens in the network, and our rules allow at most one token per place, the algorithm results in $n$ selected antennas. Executing the algorithm on several RPNs in parallel (in our experiments, up to five) allows tokens to 
traverse all parts of the network and find good configurations even with a relatively small number of antennas and users. The converged state of the RPN becomes the physical state of antennas: antennas with tokens are turned on for the duration of the coherence interval. At the next update of the channel state information, the algorithm proceeds from the current state.

The computational footprint of the described algorithm is very small: two small matrix multiplications and determinant calculations are performed at a node which contains a token in a small number of iterations. As such, this algorithm is significantly faster and computationally less demanding than the centralised greedy approach which is a low-complexity representative of global optimisation algorithms in antenna selection [11]. The worst case complexity of the RPN based approach is $\mathcal{O}\left(N_{T}^{\omega / a}\right)$ (here, $N_{T}$ denotes the number of antennas, and $\omega, 2<\omega<3$ is the exponent in the employed matrix multiplication algorithm complexity). The parameter $a$ is related to the relative size of the neighbourhood as a reciprocal exponent, assuming that a neighbourhood of $N_{T}^{1 / a}$, $a>1$ suffices for RPN algorithm (as $\sqrt{N_{T}}$ suffices in our case, we went for $a=2$ ). The constant factor multiplying the complexity is small because of few computing nodes (only those with tokens) and few iterations.

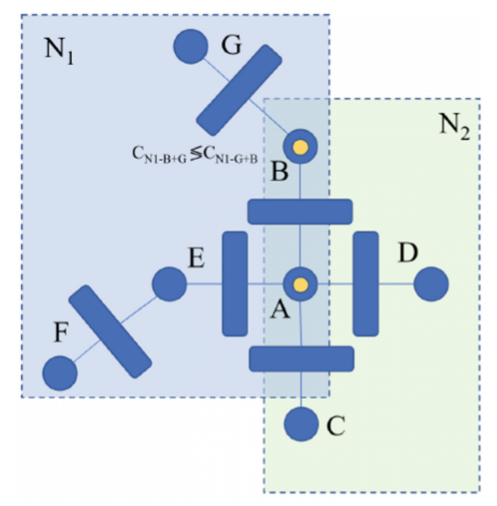

Fig. 1. A toy model of antenna selection on a reversing Petri net

\subsection{Results and Discussion}

The algorithm was tested using the raytracing Matlab tool Ilmprop [9] on a system composed of 64 omnidirectional antennas randomly distributed in space shown in Fig. 2(a). In all computations, channel state information (CSI) in matrix $\mathbf{H}$ was normalised to unit average energy over all antennas, users and subcarriers, following the practice from [10]. 75 randomly distributed scatterers and one large obstacle are placed in the area with the distributed base station. The number of (randomly distributed) users with omnidirectional antennas varied 


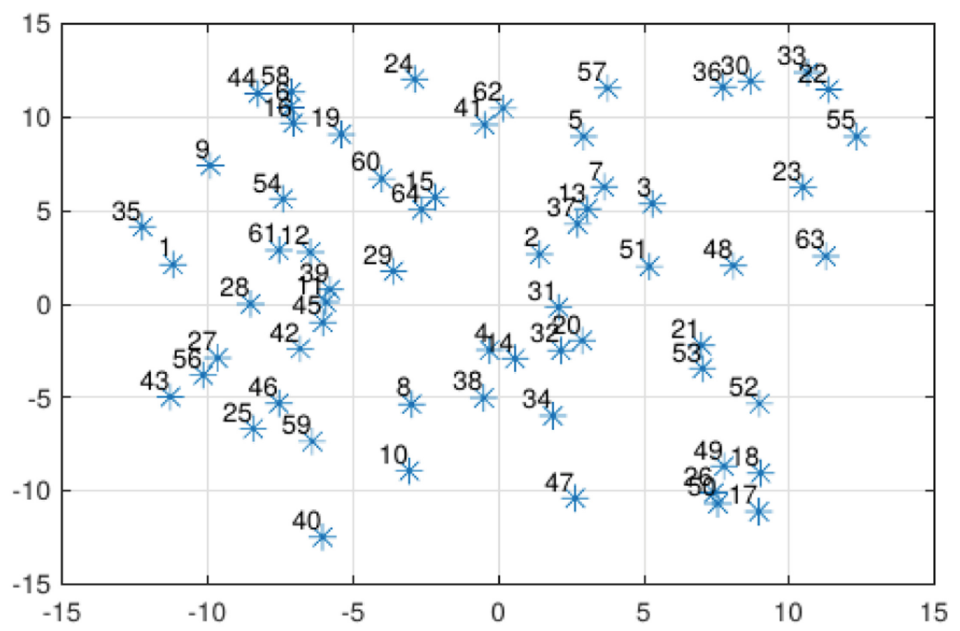

(a) Randomly distributed antennas

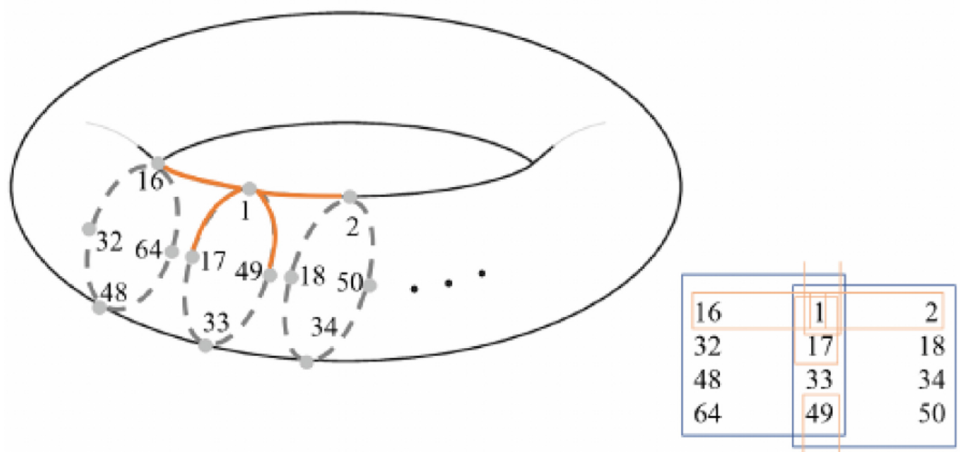

(b) The mapping to RPN topology

Fig. 2. Antennas in physical and computational domain

from 4 to 16, and we used 300 OFDM (orthogonal frequency-division multiplexing) subcarriers, SNR $\rho=-5 \mathrm{~dB}, 2.6 \mathrm{GHz}$ carrier frequency, $20 \mathrm{MHz}$ bandwidth. Antennas are computationally arranged in an $4 \times 16$ array folded into a toroid, creating a continuous infinite network, as shown in Fig. 2(b), e.g. antenna 1 is a direct neighbour of antennas 2, 16, 17 and 49. Immediate Von Neumann (top, down, left, right) neighbours can exchange tokens, and overlapping 8-antenna neighbourhoods are placed on the grid: e.g. for antenna 1, transitions to 16 and 17 are decided upon within the neighbourhood $\{16,32,48,64,1,17,33,49\}$ and the transitions to 2 and 49 are in $\{1,17,33,49,2,18,34,50\}$. In Fig. 3 we compare greedy and random selection with two variants of our RPN approach: the average of five concurrently running RPNs, and the performance of the best RPN out of those five. The performance is comparable in all cases, and both 
variants of our proposed algorithm tend to outperform the centralised approach as the number of users grows. This in practice means that a single RPN suffices for networks with a relatively large expected number of users.

The inherent reversibility of this problem and its solution generalises to the common problem of resource allocation in wireless networks, and sharing any pool of resources (power, frequency, etc.) can be handled between antennas (and antenna clusters) over a Reversing Petri Net. At the same time, such a solution would be robust to changes in the environment, potential faults, sudden changes in the mode of operation, and could operate on reversible hardware.
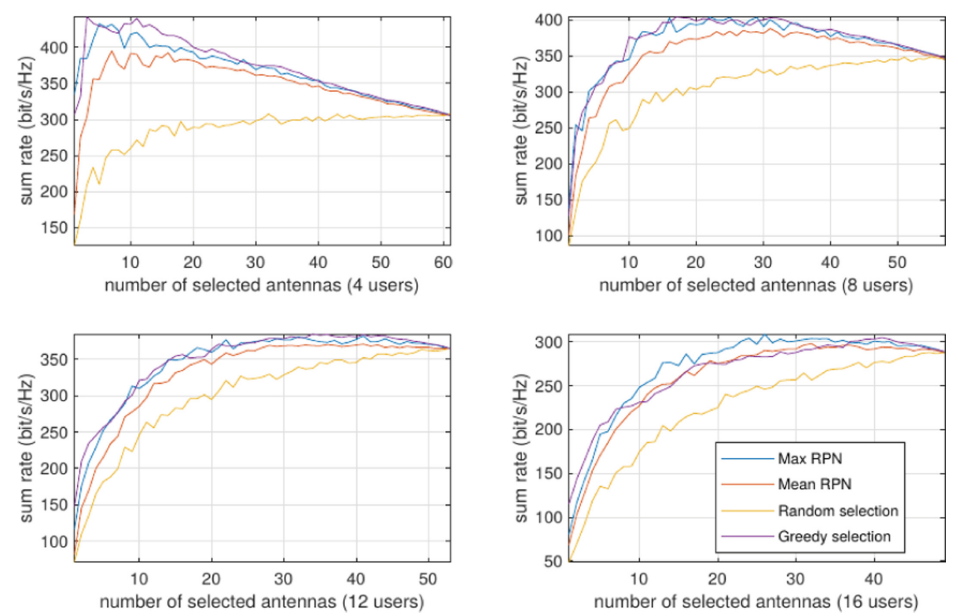

Fig. 3. Achieved sum rates for $4-16$ users using the proposed algorithm vs random and centralised greedy selection

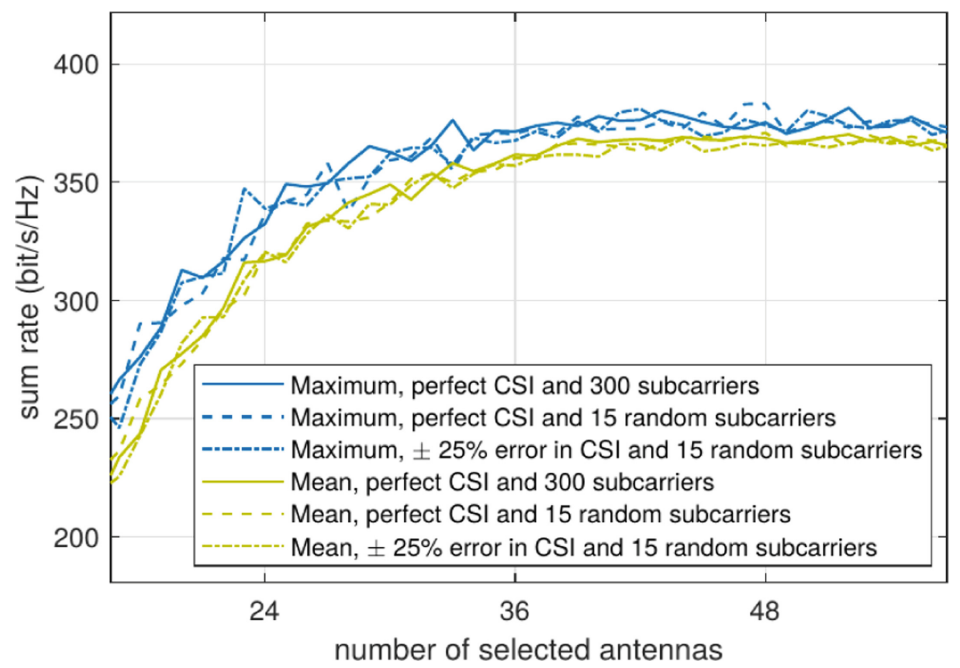

Fig. 4. The effects of imperfect CSI and random selection of subcarriers on optimisation 
In [21], it has been shown that the distributed algorithms are resistant to errors in CSI and that they perform well even with just a (randomly selected) subset of subcarriers used for optimisation. Results in Fig. 4 in the case of 12 users confirm this for the RPN algorithm as well.

\section{Reversible Hardware for Time Reversal}

The technique called wave time reversal [6] has been introduced in acoustics almost three decades ago, and has since been applied to other waves as welloptical and RF. In our work, we focused on acoustic time reversal, thinking of its applications in acoustic underwater communications. However, it is worth noting that wave time reversal plays a significant role in $\mathrm{RF}$ communications as well-conjugate beamforming for MIMO systems is based on it. In the remainder of this section, we introduce the concept of wave time reversal and explain our proposed solution for its reversible hardware implementation. The presentation here follows the one in [20].

\subsection{Wave Time Reversal}

Time reversal mirrors (TRMs) [6] are based on emitter-receptor antennas positioned on an arbitrary enclosing surface. The wave is recorded, digitised, stored, time-reversed and rebroadcasted by the same antenna array. If the array on the boundary intercepts the entire forward wave with a good spatial sampling, it generates a perfect backward-propagating copy. The procedure begins when the source radiates a wave inside a volume surrounded by a two-dimensional surface with sensors (microphones) along the surface which record the field and its normal derivative until the field disappears (Fig. 5). When this recording is emitted back, it created the time-reversed field which looks like a convergent wavefield until it reaches the original source, but from that point it propagates as a diverging wavefield. This can be compensated by an active source at the focusing point cancelling the field, or a passive sink as a perfect absorber [3].

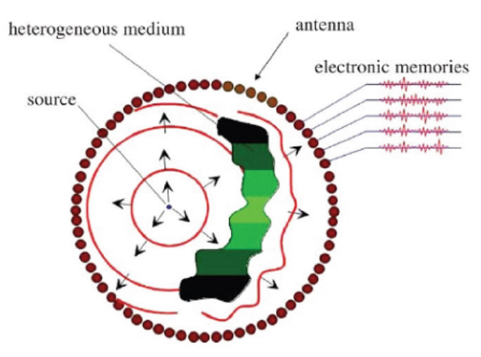

Fig. 5. A closed surface is filled with transducer elements [7]. The wavefront distorted by heterogeneities comes from a point source and is recorded on the cavity elements. The recorded signals are time-reversed and re-emitted by the elements. The timereversed field back-propagates and refocuses exactly on the initial source. 


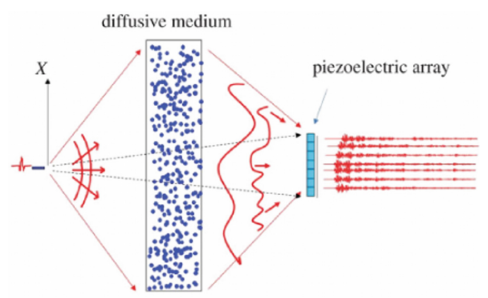

Fig. 6. Time-reversal experiment through a diffusive medium [7]

This description asks for the whole surface to be covered with the TRM transceivers, and for both the signal and the derivative to be stored: for practical purposes, less hardware-demanding solutions are needed. First, we note that the normal derivative of the field is proportional to the field in case the TRM is in the far field, halving the necessity for signal recording. Second, we note that a TRM can use complex environments to appear as an antenna wider than it is, resulting in a refocusing quality that does not depend on the TRM aperture [4]. Hence, it can be implemented with just a subset of transceivers located in one part of the boundary, as seen in Fig. 6.

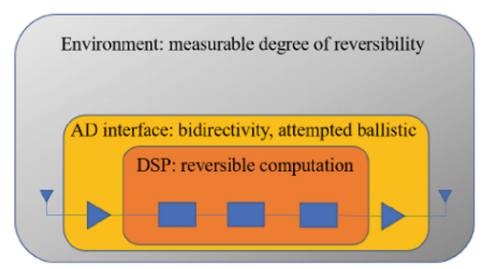

(a)

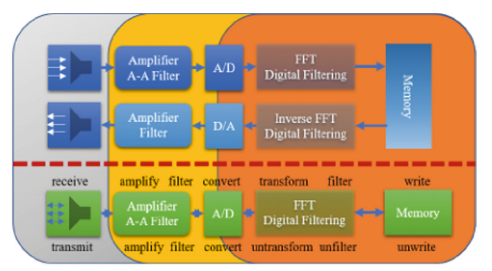

(b)

Fig. 7. (a) The three realms of reversibility, (b) The classical (top) and the reversible solution (bottom) for the classical time reversal chain

\subsection{The Design}

Figure 7 illustrates the challenge of designing a reversible hardware solution for a TRM:

1. The environment is reversible to an extent (we will return to this question later in this chapter). The physics of wave propagation in water is reversible, but issues arise as we lose information in the process.

2. The analog computation part of the TRM loses information due to filtering and analog-to-digital/digital-to-analog conversion (ADC/DAC), amplifiers accompanying the filters and the converters themselves, at the transition to the digital domain. 
3. Finally, the digital computation part of the TRM is reversible and no increase in entropy is necessary: writing in memory and unwriting, in the fashion of Bennett's trick, enabling reuse of memory for the next incoming wave, while not increasing the entropy.

Analog Processing. The real amplifier is an imperfect device with a limited bandwidth, hence prone to losing signal information. By definition, it takes additional energy for the signal, so it asks for an additional power source. At the same time, the analog to digital and digital to analog converters both lose information because of the finite resolution in time and amplitude, preventing full reversibility. However, a single device can be both an ADC and a DAC depending on the direction [14]. In this solution, we assume bi-directional converters placed together with bi-directional amplifiers [14]. The conversion is additionally simplified in the one-bit solution [5] where the receivers at the mirror register only the sign of the waveform and the transmitters emit the reversed version based on this information. It is a special case of analog-to-digital and digital-to-analog conversion with single bit converters. The reduction in discretisation levels also means simplification of the processing chain and making its reversal (bi-directivity) even simpler. The question of the information loss is not straightforward: while the information about the incoming wave is lost in the conversion process (and the loss is maximal due to minimal resolution), spatial and temporal resolution are not significantly degraded. This scheme can also be called "one-trit" (trit is a ternary digit, analogous to a bit) reversal: there are three possible states in the practical implementation: positive pressure, negative pressure, and "off".

Digital Processing. The first, straightforward way of performing time reversal of a digitally sampled wave is storing it in memory and reading the samples in the reverse order (last in, first out, LIFO), analogous to storing the samples on the stack. The design of registers in reversible logic is a well-explored topic [15] and both serial and parallel reading/writing can be implemented. Design of latches in reversible logic is a well-studied problem with known solutions; a combination of latches makes a flip-flop, and a series of flip-flops makes a register (and a reversible address counter). In the case of wave time reversal, the recording of data is a large register being loaded serially with wave data. $m$ bits from the ADC are memorised at the converter's sample rate inside a $k \times m$ bit register matrix (where $k$ is the number of samples to be stored for time reversal). In the receiving process the bits are stored, in the transmission process they are unstored, returning the memory into the blank state it started from (uncomputation). We utilise Bennett's trick and lose information without the entropic penalty: the information is kept as long as it is relevant.

When additional signal processing, e.g. filtering or modulation is performed, it is convenient to reverse waves in the frequency domain: there, time domain reversal is achieved by phase conjugation, i.e. changing the sign of the signal's phase. The transition from the time to the frequency domain (and vice versa) in the digital domain is performed by the Fast Fourier Transform (FFT) and 
its inverse counterpart, which are reversibly implementable [23]. The necessary phase conjugation is an arithmetic operation of sign reversal, again reversible. Any additional signal processing can be reversible as well: e.g. filter banks and wavelet transforms. These processes remain reversible with preservation of all components of signals [2].

Figure 8(a) gives a comparison of the bit erasures in different implementations of the digital circuitry: frequency domain (FFT) and time domain reversal performed by irreversible circuits, compared to reversible implementations. The number of erasures changes depending on two parameters: bit resolution of the $\mathrm{ADC}$ and the waiting time-the length of the interval in which samples are collected before reversal starts, equivalent to the number of digitised samples. The increase in both means additional memory locations and additional dissipation for irreversible circuits. The irreversible FFT implementation has an additional information loss caused by additional irreversible circuitry compared to the irreversible time domain implementation. Our implementation has no bit erasures whatsoever. The price that is paid reflects in the larger number of gates used in the circuit: the number of gates has only spatial consequences, informationrelated energy dissipation is zero thanks to information conservation.

On the other hand, Fig. 8(b) shows the information loss in the analog part of the system, and we differentiate two typical environments, the chaotic cavity and the complex (multiple scattering) medium. The chaotic cavity is an ergodic space with sensitive dependence on initial conditions for waves. In such an environment there is little to no loss in the information if the waiting time is long enough and the ADC resolution is high enough. In the complex media, the difference is caused by some of the wave components being reflected backwards by the scattering environment, hence not reaching the TRM. Again, more information is retained with the increase in the ADC resolution. However, as reported in [5], the information loss from low-resolution ADC use does not affect the performance of the algorithm. The analog part of the scheme remains a topic of our future work, as it leaves space for improvements of the scheme.

\section{Reversible Environment Models and Control}

Time reversal described in the previous section is an example of a reversible process in a nominally reversible environment. While dynamics of water subject to waves are inherently reversible, most of the sources of the water dynamics do not reverse naturally: e.g. the Gulf stream or a motion of a school of fish. Hence, even though it would rarely be completely reversed, the model for UAC should be reversible. We discuss the questions of reversible models following the exposition in [19], and the work in progress on control of reversible cellular automata (RCA).

RCA lattice gas models are cellular automata obeying the laws of fluid dynamics described by the Navier-Stokes equation. One such model, FHP (Frisch- Hasslacher-Pomeau) lattice gas [8] is simple and yet following the NavierStokes equations exactly. It is defined on a hexagonal grid with the rules of particle collision shown in Fig. 9. The FHP lattice gas provides us a two-dimensional 


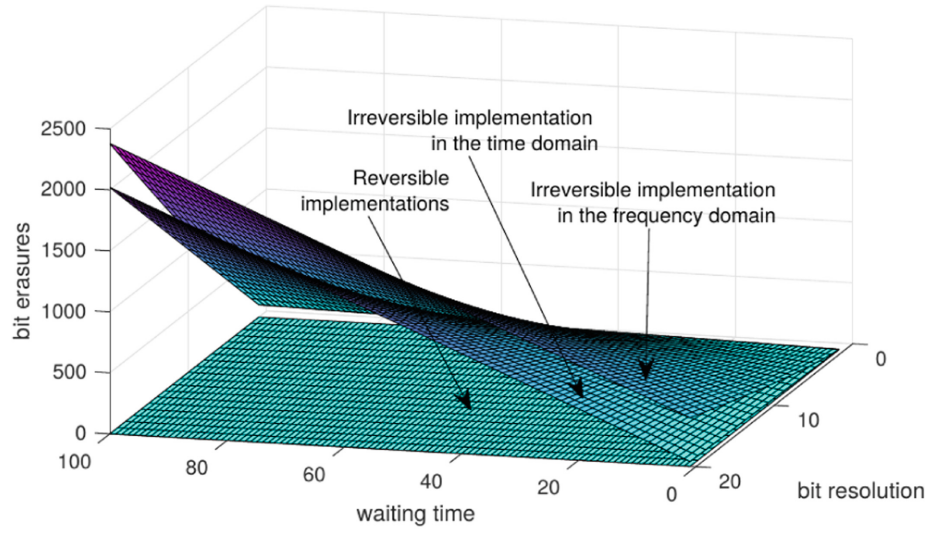

(a)

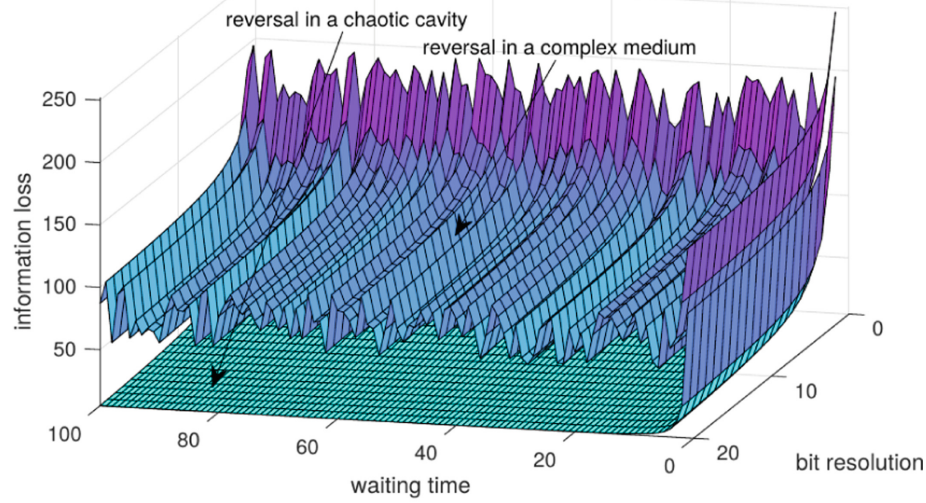

(b)

Fig. 8. Information loss in (a) the digital and (b) the analog part of the system. Units are omitted as the particular aspects of implementation are not relevant for the illustration of effects. Plot (a) is obtained by counting operations, plot (b) by simulation of back-scattering.
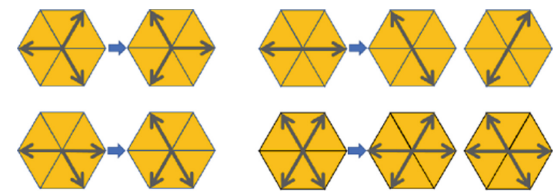

Fig. 9. FHP rules 
model for UAC, easily implementable in software and capturing the necessary properties of the reversible medium.

Following the exposition in the previous section, we observe a model with an original source (transmitter) which causes the spread of an acoustic wave, the original sink (receiver) waiting for the wave to reach it, as well as scatterers and constant flows (streams) in the environment. The constant stream and the loss of information caused by some wave components never reaching the sink will result in an imperfect reversal at the original source. The measure of returned power gives us a directivity pattern (focal point). The amplitude of the peak will fluctuate based on the location of the original source and is a measure of reversibility, akin to fidelity or Loschmidt Echo. For us, it is a measure of the quality of communication, but in a more general context it can measure reversibility of a cellular automaton.

From the control viewpoint, it is interesting to ask the following: if a certain part of the environment is controllable (i.e. a number of cells of the RCA does not obey the rules of the RCA but allows external modification), how can it be used to achieve better time reversal? This is a compensation approach where we engineer the environment to compensate for effects caused by sources of disturbance out of our control. The approach we take is one of control of cellular automata [1], and it is expected that RCA are easier to control than regular CA, with easier search strategies and the ability to calculate control sequences.

\section{Conclusions}

In this chapter, we provided an overview of results obtained in the case study on reversible computation in wireless communications. Some of the presented work, such as optimisation in massive MIMO and reversible hardware for wave time reversal is finished and subject to further extensions and generalisations; other work, mainly the parts focused on RCA and modelling of reversible physics of communication, is still ongoing and more results are to come. This has been a pioneering study into reversibility in communications, and the results obtained promise a lot of space for improvement and applications in the future. We hope these efforts will serve as an inspiration and a trigger for the development of this field of research.

Acknowledgements. The work presented in this chapter was supported by the COST Association through the IC1405 Action on Reversible Computation, as well as a grant from Science Foundation Ireland (SFI) co-funded under the European Regional Development Fund under Grant Number 13/RC/2077 and European Union's Horizon 2020 programme under the Marie Skłodowska-Curie grant agreement No 713567. I am grateful to my collaborators, Prof Anna Philippou, Kyriaki Psara, Dr Julien de Rosny, Prof Mathias Fink, and Dr Franco Bagnoli for making this interdisciplinary research possible, and to Konstantin Popovic for the inspiring ideas. 


\section{References}

1. Bagnoli, F., Rechtman, R., El Yacoubi, S.: Control of cellular automata. Phys. Rev. E 86(6), 066201 (2012)

2. Chen, Y.-J., Amaratunga, K.S.: M-channel lifting factorization of perfect reconstruction filter banks and reversible M-band wavelet transforms. IEEE Trans. Circ. Syst. II Analog Digit. Signal Process. 50(12), 963-976 (2003)

3. de Rosny, J., Fink, M.: Overcoming the diffraction limit in wave physics using a time-reversal mirror and a novel acoustic sink. Phys. Rev. Lett. 89(12), 124301 (2002)

4. Derode, A., Roux, P., Fink, M.: Robust acoustic time reversal with high-order multiple scattering. Phys. Rev. Lett. 75(23), 4206 (1995)

5. Derode, A., Tourin, A., Fink, M.: Ultrasonic pulse compression with one-bit time reversal through multiple scattering. J. Appl. Phys. 85(9), 6343-6352 (1999)

6. Fink, M.: Time reversal of ultrasonic fields. I. Basic principles. IEEE Trans. Ultrason. Ferroelectr. Freq. Control 39(5), 555-566 (1992)

7. Fink, M.: From Loschmidt daemons to time-reversed waves. Philos. Trans. Roy. Soc. A Math. Phys. Eng. Sci. 374(2069), 20150156 (2016)

8. Frisch, U., Hasslacher, B., Pomeau, Y.: Lattice-gas automata for the Navier-Stokes equation. Phys. Rev. Lett. 56(14), 1505 (1986)

9. Del Galdo, G., Haardt, M., Schneider, C.: Geometry-based channel modelling of MIMO channels in comparison with channel sounder measurements. Adv. Radio Sci. 2(BC), 117-126 (2005)

10. Gao, X., Edfors, O., Tufvesson, F., Larsson, E.G.: Massive MIMO in real propagation environments: do all antennas contribute equally? IEEE Trans. Commun. 63(11), 3917-3928 (2015)

11. Gao, Y., Vinck, H., Kaiser, T.: Massive MIMO antenna selection: switching architectures, capacity bounds, and optimal antenna selection algorithms. IEEE Trans. Signal Process. 66(5), 1346-1360 (2017)

12. Gharavi-Alkhansari, M., Gershman, A.B.: Fast antenna subset selection in MIMO systems. IEEE Trans. Signal Process. 52(2), 339-347 (2004)

13. Hoydis, J., Ten Brink, S., Debbah, M.: Massive MIMO in the UL/DL of cellular networks: how many antennas do we need? IEEE J. Sel. Areas Commun. 31, 160 $171(2013)$

14. Mirmotahari, O., Berg, Y.: Pseudo floating-gate and reverse signal flow. In: Recent Advances in Technologies. IntechOpen (2009)

15. Nayeem, N.M., Hossain, M.A., Jamal, L., Babu, H.M.H.: Efficient design of shift registers using reversible logic. In: 2009 International Conference on Signal Processing Systems, pp. 474-478. IEEE (2009)

16. Ozgur, A., Lévêque, O., Tse, D.: Spatial degrees of freedom of large distributed MIMO systems and wireless ad hoc networks. IEEE J. Sel. Areas Commun. 31(2), 202-214 (2013)

17. Philippou, A., Psara, K.: Reversible computation in petri nets. In: Kari, J., Ulidowski, I. (eds.) RC 2018. LNCS, vol. 11106, pp. 84-101. Springer, Cham (2018). https://doi.org/10.1007/978-3-319-99498-7_6

18. Philippou, A., Psara, K., Siljak, H.: Controlling reversibility in reversing petri nets with application to wireless communications. In: Thomsen, M.K., Soeken, M. (eds.) RC 2019. LNCS, vol. 11497, pp. 238-245. Springer, Cham (2019). https://doi.org/ 10.1007/978-3-030-21500-2_15 
19. Siljak, H.: Reversibility in space, time, and computation: the case of underwater acoustic communications. In: Kari, J., Ulidowski, I. (eds.) RC 2018. LNCS, vol. 11106, pp. 346-352. Springer, Cham (2018). https://doi.org/10.1007/978-3-31999498-7_25

20. Siljak, H., de Rosny, J., Fink, M.: Reversible hardware for acoustic communications. IEEE Commun. Mag. 58, 55-61 (2020)

21. Siljak, H., Macaluso, I., Marchetti, N.: Distributing complexity: a new approach to antenna selection for distributed massive MIMO. IEEE Wireless Commun. Lett. 7(6), 902-905 (2018)

22. Siljak, H., Psara, K., Philippou, A.: Distributed antenna selection for massive MIMO using reversing Petri nets. IEEE Wireless Commun. Lett. 8(5), 1427-1430 (2019)

23. Skoneczny, M., Van Rentergem, Y., De Vos, A.: Reversible Fourier transform chip. In: 2008 15th International Conference on Mixed Design of Integrated Circuits and Systems, pp. 281-286. IEEE (2008)

24. Vaze, R., Ganapathy, H.: Sub-modularity and antenna selection in MIMO systems. IEEE Commun. Lett. 16(9), 1446-1449 (2012)

Open Access This chapter is licensed under the terms of the Creative Commons Attribution 4.0 International License (http://creativecommons.org/licenses/by/4.0/), which permits use, sharing, adaptation, distribution and reproduction in any medium or format, as long as you give appropriate credit to the original author(s) and the source, provide a link to the Creative Commons license and indicate if changes were made.

The images or other third party material in this chapter are included in the chapter's Creative Commons license, unless indicated otherwise in a credit line to the material. If material is not included in the chapter's Creative Commons license and your intended use is not permitted by statutory regulation or exceeds the permitted use, you will need to obtain permission directly from the copyright holder.

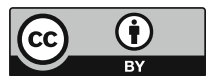

